\title{
Higher Tori in Volume-Preserving Maps
}

\author{
Gerold Baier \\ Institute for Chemical Plant Physiology \\ Michael Klein and Otto E. Rössler \\ Institute for Physical and Theoretical Chemistry, University of Tübingen, Tübingen, FRG
}

Z. Naturforsch. 45a, 664-668 (1990); received January 16, 1990

A hierarchy of $n$-Tori is demonstrated numerically in volume-preserving $(n+1)$-dimensional invertible maps.

Key words: Volume-preserving maps, torus, hypertorus, attracting torus, chaos,

\section{Introduction}

A hierarchy of chaotic and mixed chaotic and toroidal attractors as a function of the number of variables involved has been proposed to exist in dissipative ODEs [1]. A number of experimental examples of three-frequency dynamics has been reported [2]. In the Belousov-Zhabotinsky reaction, experimental and theoretical evidence for a type of behavior has been presented where the system switches autonomously between two types of toroidal oscillations $[3,4]$. On reducing the dimension of the flow by means of a Poincare cross-section, an attracting closed loop is found in the case of a toroidal flow. Similarly, the cross-section of an ideal three-torus covers the surface of a two-torus. In the modelling of experimental systems it is desirable to have access to a set of prototypic maps showing higher types of toroidal and mixed toroidal and chaotic solutions, since explicit maps are easier to investigate than four-variable nonlinear ODEs.

We apply a building-blocks design principle to volume-preserving maps to generate closed loops, twotori, and three-tori, respectively. We then construct a volume-contracting version of the map and demonstrate a toroidal attracting set in a diffeomorphic region of the 3-D map. We then study distortions of the torus as a function of parameters.

Reprint requests to G. Baier, Institut für Chemische Pflanzenphysiologie, Universität Tübingen, Corrensstr. 41, D-7400 Tübingen, FRG.

\section{Results}

Iterative invertible mappings can exhibit nontrivial asymptotic dynamics as a function of the parameters. A precondition for arriving at a stable attracting set is that the phase space volume contract under iteration. This can be controlled by means of the Jacobian. An invertible map is dissipative (volume-contracting) if the absolute value of the Jacobian is smaller than one. A special case of stable dynamics are volume-preserving and orientation-preserving maps, with a Jacobian of unity. Whereas in the dissipative case the attracting set is reached independently of the initial conditions when one starts inside the basin of attraction, in the volume-preserving case the dynamics remains a function of the initial conditions. We investigate such mappings in the case of two, three, and four variables.

The map

$$
\begin{aligned}
& x_{n+1}=A+B x_{n}-y_{n}, \\
& y_{n+1}=x_{n}
\end{aligned}
$$

is a discrete two-variable system with Jacobian equal to 1 . This system yields a period four dynamics as long as $B=0$. The sequence is: $x_{0}, A-y_{0}, A-x_{0}$, and $y_{0}$ for $x$ with initial conditions $x_{0}$ and $y_{0}$. For $B=1$, one finds a similar sequence of period six $\left(x_{0}, A+x_{0}-y_{0}\right.$, $2 A-y_{0}, 2 A-x_{0}, A-x_{0}+y_{0}$ and $y_{0}$ for $x$ ). The characteristic equation is equal to unity for $\lambda_{1}=0$ and $\lambda_{2}=B$. This means that for $0<B<1$ there exists a family of closed loops for all initial conditions.

0932-0784 / 90 / 0500-0664 \$ 01.30/0. - Please order a reprint rather than making your own copy. 
Now consider the following extension:

$$
\begin{aligned}
& x_{n+1}=A+B x_{n}-C y_{n}+D z_{n}, \\
& y_{n+1}=x_{n}, \\
& z_{n+1}=y_{n}+E-F x_{n}^{2} .
\end{aligned}
$$

With $D=1$, the map is 3-dimensional and volume-preserving for arbitrary values of $A, B, C, E$, and $F$. The variable $y$ always follows $x$ with one delay step, so it is sufficient to prevent $x$ from exploding by means of a quadratic term, $-F x_{n}^{2}$. The system is stable with period four when $B=D=0$. For $D>0$ we find toroidal behavior. Figure 1 shows one out of a nested family of stable toroidal results for a set of parameters and initial conditions. The surface of the two-torus is filled with points, the dynamics is presumably quasiperiodic. Because of the nonlinear term only a subset of all possible initial conditions shows stable solutions: $z$ explodes for large initial values. Incidentally, a hypertoroidal flow governed by a similar map as a crosssection has been described for a four-variable volumepreserving ordinary differential equation [5].

A hypertoroidal dynamics can be obtained from the present three-variable map by means of introducing a second delay variable:

$$
\begin{aligned}
& x_{n+1}=A+B x_{n}-C y_{n}+D z_{n}-w_{n}, \\
& y_{n+1}=x_{n}, \\
& z_{n+1}=y_{n}+E-F x_{n}^{2}, \\
& w_{n+1}=z_{n} .
\end{aligned}
$$

A numerical result for parameter values that proved stable under a large number of iterations is shown in Figure $2 \mathrm{a}$. To analyze the structure of this complicated toroidal motion we show a cut of the three-torus in Figure $2 \mathrm{~b}$. The cut looks like a torus, the points cover a two-dimensional surface (actually, there are two torus-shaped cuts placed one above each other).

Finally, we destroy the conservativity condition by introducing a nonlinear mixed term. Substituting the term $y_{n}$ by $x_{n} y_{n}$ in the first line of (1) we obtain a well-known endomorphism, the delayed logistic map [6]. Here an attracting closed loop is found beyond the (primary) Neimark bifurcation of the steady state ( $B=1.0$ and $A>1)$. The previous family of invariant closed loops contracts to an asymptotic set of the same topology. Similarly in the $3 \mathrm{D}$ case, by substituting $B x z$ for $B x$ in the first line of (2), a no longer everywhere diffeomorphic map (with Jacobian 1+Bx)

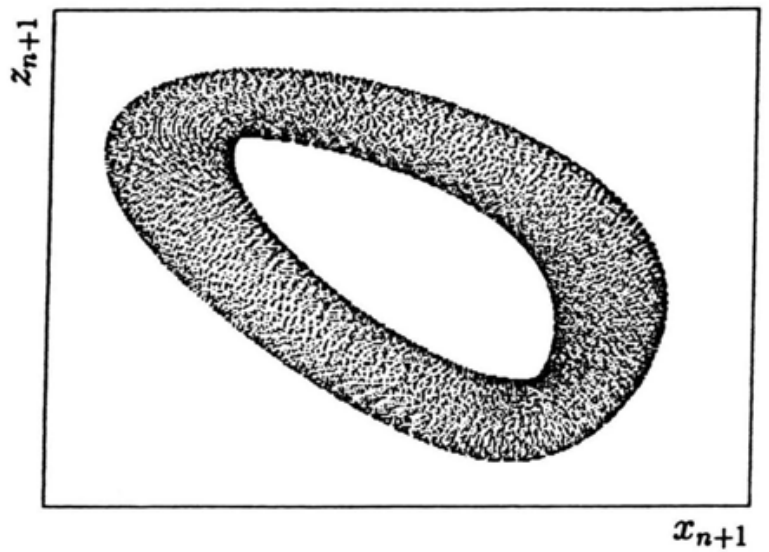

Fig. 1. Numerical simulation of (2) with $A=B=F=0.2$, $C=0.0, E=-0.2, D=1.0$ and initial conditions $x_{0}=1.0$, $y_{0}=0.1, z_{0}=0.0$. Axes: $-0.6 \ldots 1.6$ for $x_{n+1}$ and $-1.0 \ldots 1.4$ for $z_{n+1}$.
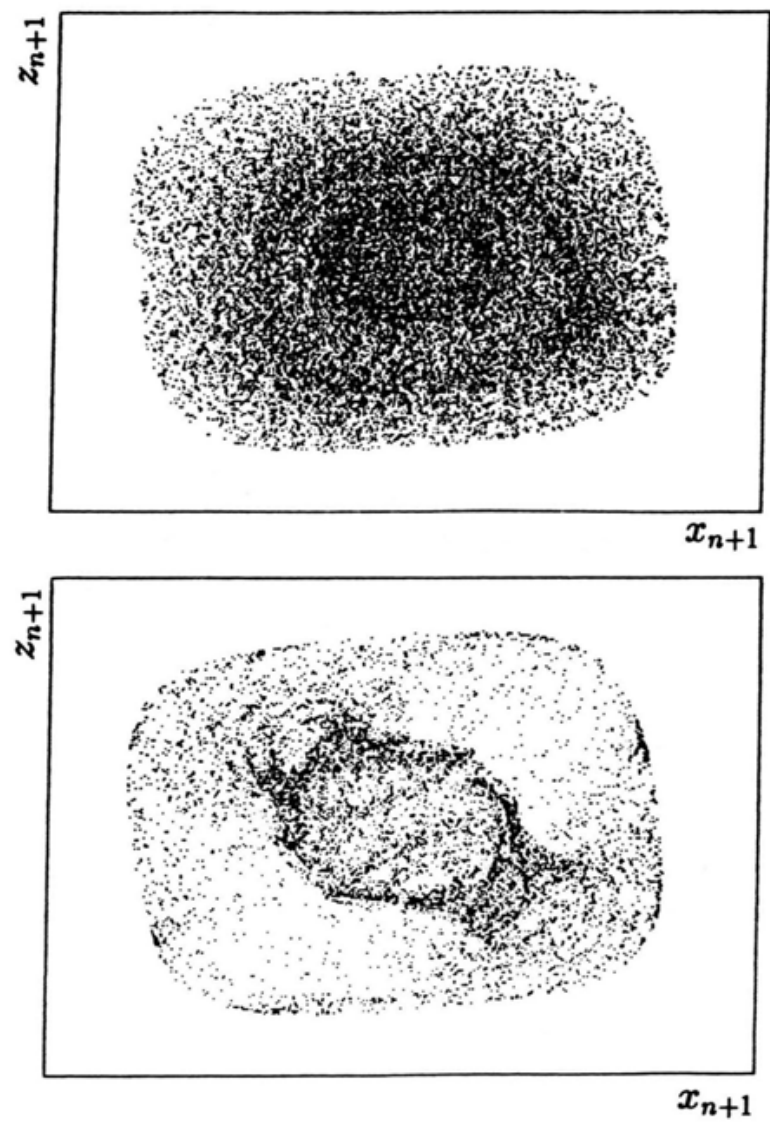

Fig. 2. a) Numerical simulation of (3). $A=-0.1, B=E=F=0.1$, $C=0.0, D=0.1$, and initial conditions $x_{0}=-0.9046$, $y_{0}=0.4326, z_{0}=0.8459, w_{0}=-1.9360$. Axes: $-5.0 \ldots 5.0$ for $x_{n+1}$ and $z_{n+1}$. b) Section through the structure in a) at $y=0 \pm 0.005,5,000,000$ iterations. 

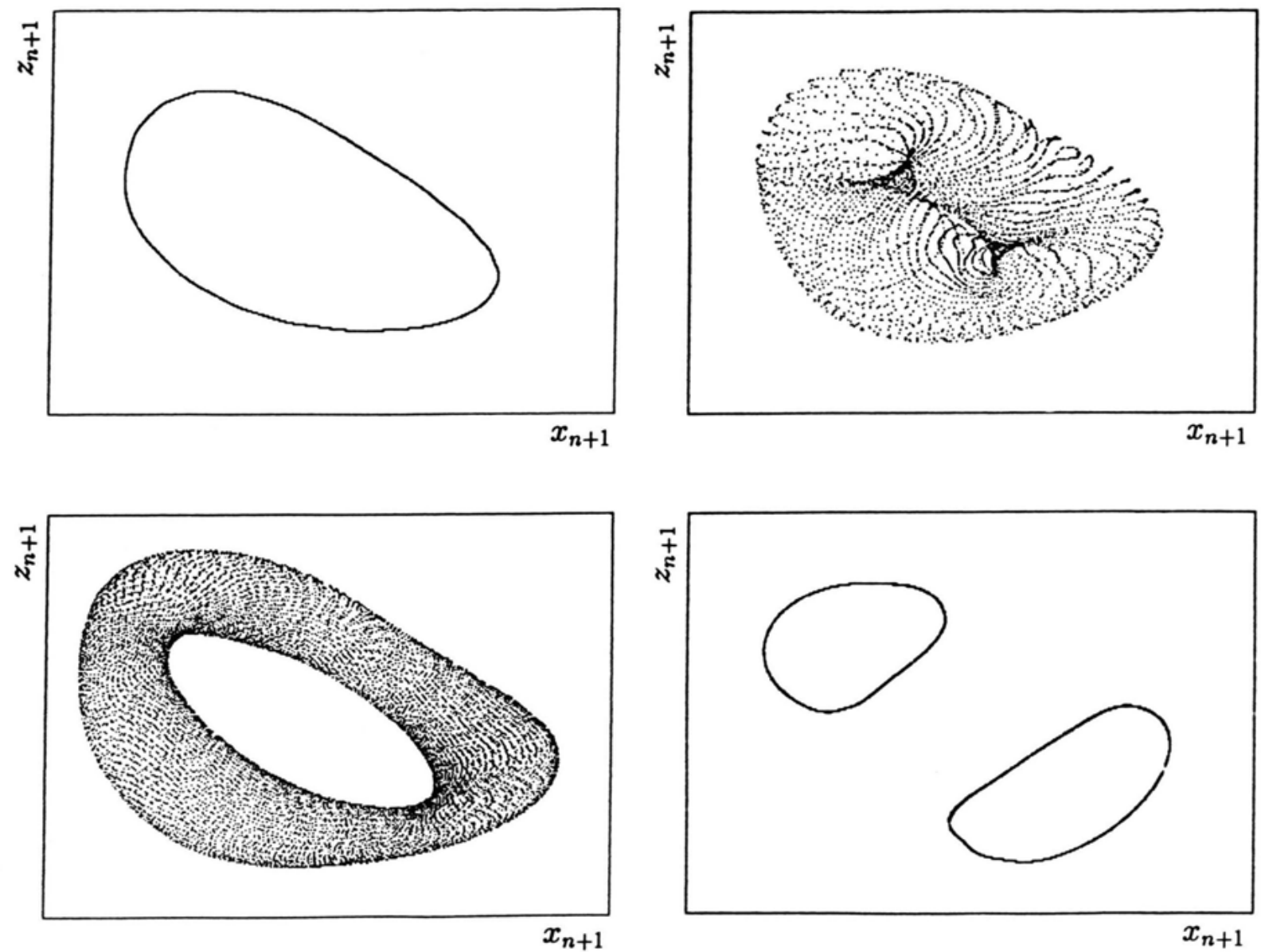

Fig. 3. Numerical simulation of (4). $A=B=F=0.2, D=1.0$, $E=0.0$. Axes: $-1.6 \ldots 2.0$ for $x_{n+1}$ and $-2.0 \ldots 2.0$ for $z_{n+1}$. a) $C=-0.03$ and initial conditions $x_{0}=-0.554, y_{0}=1.253$, $z_{0}=-0.8266$. b) $C=-0.042$ and initial conditions $x_{0}=$ $-0.0216, y_{0}=-1.2635, z_{0}=0.6392$.

is created:

$$
\begin{aligned}
& x_{n+1}=A+B x_{n} z_{n}-C y_{n}+D z_{n}, \\
& y_{n+1}=x_{n}, \\
& z_{n+1}=y_{n}+E-F x_{n}^{2} .
\end{aligned}
$$

For the set of parameters given in the legend of Fig. 3, we find an attracting closed loop for $C<C_{c} \approx 0.039$ (Figure $3 \mathrm{a}$ ). For $C=C_{c}$ this loop undergoes a bifurcation. For $C>C_{c}$ an attracting two-torus is created. In analogy to the situation valid in the delayed logistic map we call this a "secondary" Neimark bifurcation (from closed loop to torus). Figure $3 \mathrm{~b}$ shows a simulation of the resulting attractor.

Fig. 4. Numerical simulation of (4). $A=B=0.2, C=-0.05$, $D=1.0, E=0.0, F=0.20$ and initial conditions $x_{0}=-0.3814$, $y_{0}=-0.1682, z_{0}=0.6476$. Axes: $-2.5 \ldots 3.0$ for $x_{n+1}$ and $-3.0 \ldots 3.0$ for $z_{n+1}$. a) Attractor. b) Cut at $y=0 \pm 0.005$. $1,500,000$ iterations.

The former closed loop has lost its stability in the same way as a limit cycle in a flow does: two "Floquet multipliers" have crossed the unit circle at complex conjugate values, cf. [6]. There exists a basin of attraction within which all initial conditions are asymptotically attracted to the surface of the torus, and the whole surface is covered quasiperiodically.

An increase of the nonlinearity $F$ leads to a distortion of the torus; lockings in the form of periodic orbits; closed loops winding along the torus; and transition to chaos. Figures $4-6$ present a sequence of three attractors together with cuts at $y_{n}=0$ on increasing the parameter $F$. In Fig. 4 , at $F=0.2$, the surface of the torus will be filled densely for a large number of iterations, 

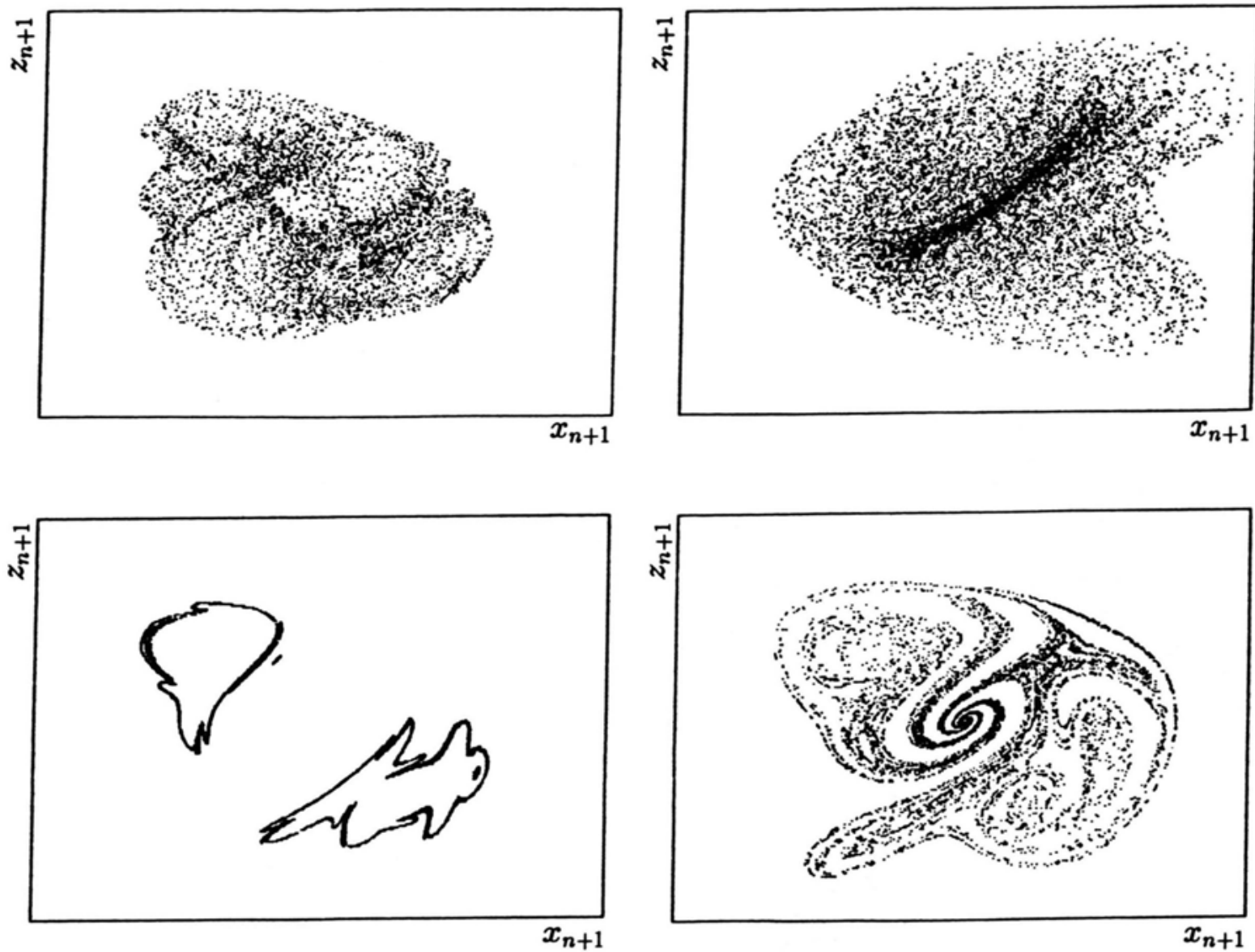

Fig. 5. Numerical simulation of (4). Same as Fig. 4, except that $F=0.25$. a) Attractor. b) Cut at $y=0 \pm 0.005$. $1,500,000$ iterations.

as can be seen from the two closed loops in the cut. In Fig. 5, at $F=0.25$, the torus is distorted leading to stretching and folding and thus chaotic dynamics. The cut shows typical "noses" which indicate the underlying Cantor set structure of the attractor. In Fig. 6, at $F=0.33$, the torus has broken up, giving rise to a higher type of chaos in the map. The cut shows a complicated distribution of points, yet some underlying structure is discernible. A "focus-like" structure is especially striking. A magnification reveals an empty central region, however, with three surrounding branches. The formerly smooth torus now appears to be composed of several entangled layers spiralling out of the center. Whether or not a second positive Lyapunov exponent can be found for this chaotic attractor is presently open.
Fig. 6. Numerical simulation of (4). Same as Fig. 4, except that $F=0.33$. a) Attractor. b) Cut at $y=0 \pm 0.005$. 5,000,000 iterations.

\section{Discussion}

We have demonstrated a procedure for systematically creating a three-torus in a four-dimensional volume-preserving map. This procedure can presumably be iterated. A hierarchy of $n$-tori seems to exist in an $n+1$-dimensional state space. Numerically, however, it becomes more difficult to find stable nonescaping solutions. This fact may point to a decreasing probability to find such high dimensional nonchaotic behavior in conservative experimental systems.

There exists a connection between volume-preserving maps and dissipative diffeomorphisms. A closed loop that appears in a $2 \mathrm{D}$ volume-preserving map can arise as an attracting set via a Neimark bifurcation. Similarly, a two-torus can be realized in $3 \mathrm{D}$ maps, 
both volume-preserving and dissipative. The state space structure of a volume-preserving map can be transformed into an attracting set by one's adding a further nonlinearity. So far, no globally invertible examples (constant Jacobian <1) have been found in our class.

In analogy to the case of systems of coupled ordinary differential equations [5], where a volume-preserving oscillator can be extended into a two-torus by means of an amplitude modulation in an additional variable, maps have been presented for which the same principle holds true. The source of this modulation in each case is the oscillation itself, and the amplitude is kept bounded within a finite size by means of a nonlinearity. This very principle could be applied twice in the map by adding another delay variable. The resulting equation creates the surface of a threetorus. The three-torus is demonstrated by means of cuts of the object in state space that yield a two-torus in a $3 \mathrm{D}$ cut (Figure $2 \mathrm{~b}$ ). Introducing one more nonlinear term of "mixed" type is then sufficient to create dissipative maps with attractors of the same topology

[1] O. E. Rössler, Z. Naturforsch. 38 a, 788 (1983).

[2] J. B. Gollub and S. V. Benson, J. Fluid Mech. 100, 449 (1979). - J. Maurer and A. Libchaber, J. Phys. Lett. 41, L515 (1980). - A. Libchaber, S. Fauve, and C. Laroche, Physica 7D, 73 (1983). - S. Martin, H. Leber, and W. Martienssen, Phys. Rev. Lett. 53, 303 (1984). - P. S. Linsay and A. W. Cumming, Physica 40 D, 196 (1989).

[3] G. Baier, J. L. Hudson, and K. Wegmann, Phys. Lett. A 141, 340 (1989).

[4] D. Barkley, J. Ringland, and J. S. Turner, J. Chem. Phys. 87, 3812 (1987). as in the volume-preserving case. The resulting equation in the case of three variables is an explicit model of a Poincaré cross-section of a hypertoroidal dissipative flow. Related results on dissipative systems were gained from numerical studies of coupled modulo maps by Grebogi et al. [7] and Kaneko [8]. Further study of hypertoroidal maps will help classifying experimental results with three independent frequencies mentioned in the introduction and will allow conjectures concerning the types of chaos and the transitions to chaos occurring on a distorted hypertorus.

An open question remains the possibility of a complete fractalization of the toroidal surface over an open-dense region of parameter space. So far only fractalized closed loops appear to be known in maps at singular points in parameter space [8]. A nowhere differentiable 2-D attractor has been described by Grebogi et al. in an invertible dissipative map which was, however, non-generic [9].

We thank Jack Hudson for discussions. G. B. thanks Klaus Wegmann for stimulation and support.

[5] O. E. Rössler, Ann. N. Y. Acad. Sci. 316, 376 (1979).

[6] J. M. Thompson and H. B. Stewart, Nonlinear Dynamics and Chaos, John Wiley and Sons, Chichester 1986.

[7] C. Grebogi, E. Ott, and J. A. Yorke, Phys. Rev. Lett. 51, 339 (1983).

[8] K. Kaneko, Collapse of Tori in Dissipative Systems, World Scientific, Singapore 1986.

[9] C. Grebogi, E. Ott, and J. A. Yorke, Physica 7D, 181 (1983). 